Address correspondence to: Sidney A. Simon, Department of Neurobiology, Duke University Medical Center, 327C Bryan Research Building, Box 3209, Durham, North Carolina 27708, USA. Phone: (919) 684-4178; Fax: (919) 684-4431; E-mail: sas@neuro.duke.edu.

1. Cechetto, D.F., Standaert, D.G., and Saper, C.B. 1985. Spinal and trigeminal projections to the parabarchial nucleus of the rat. J. Comp. Neurol. 240:153-160.

2. Lundberg, J.M., and Saria, A. 1983. Capsaicininduced desensitization of airway mucosa to cigarette smoke, mechanical and chemical irritants. Nature. 302:251-253.

3. Geppetti, P., et al. 1993. Ruthenium red. Br. J. Pharmacol. 108:646-650.

4. Andrè, E., et al. 2008. Cigarette smoke-induced neurogenic inflammation is mediated by $\alpha, \beta$ unsaturated aldehydes and the TRPA1 receptor in rodents. J. Clin. Invest. 118:2574-2582.

5. Nassenstein, C., et al. 2008. Expression and function of the ion channel TRPA1 in vagal afferent nerves innervating mouse lungs. J. Physiol. (Lond.) 586:1595-1604.

6. Nassenstein, C., et al. 2008. Expression and function of the ion channel TRPA1 in vagal afferent nerves innervating mouse lungs. J. Physiol. 586:1595-1604.

7. Salazar, H., et al. 2008. A single N-terminal cysteine in TRPV1 determines activation by pungent compounds from onion and garlic. Nat. Neurosci. 11:255-261.

8. Macpherson, L.J., et al. 2005. The pungency of garlic: activation of TRPA 1 and TRPV1 in response to allicin. Curr. Biol. 15:929-934.

9. Maher, M., et al. 2008. Activation of TRPA1 by farnesyl thiosalicylic acid. Mol. Pharmacol. 73:1225-1234.

10. Hinman, A., Chuang, H., Bauitsta, D.M., and Julius, D. 2006. TRP channel activation by reversible covalent modification. Proc. Natl. Acad. Sci. U. S. A. 103:19564-19568.

11. Calixto, J.B., Kassuya, C.A.L., André, E., and Ferreira, J. 2005. Contribution of natural products to the discovery of the transient receptor potential (TRP) channels family and their functions. Pharmacol. Ther. 106:179-208.

12. Bautista, D.M., et al. 2006. TRPA1 mediates the inflammatory actions of environmental irritants and proalgesic agents. Cell. 124:1269-1282.

13. Niforatos, W., et al. 2007. Activation of TRPA1 channels by the fatty acid amide hydrolase inhibitor 3'-carbamoylbiphenyl-3-yl cyclohexylcarbamate (URB597). Mol. Pharmacol. 71:1209-1216.

14. Merrill, A.W., Cuellar, J.M., Judd, J.H., Carstens, M.I., and Carstens, E. 2008. Effects of TRPA1 agonists mustard oil and cinnamaldehyde on lumbar spinal wide-dynamic range neuronal responses to innocuous and noxious cutaneous stimuli in rats. J. Neurophysiol. 99:415-425.

15. Trevisani, M., et al. 2007. 4-Hydroxynonenal, an endogenous aldehyde, causes pain and neurogenic inflammation through activation of the irritant receptor TRPA1. Proc. Natl. Acad. Sci. U. S. A. 104:13519-13524.

16. Bessac, B.F., et al. 2008. TRPA1 is a major oxidant sensor in murine airway sensory neurons. J. Clin. Invest. 118:1899-1910.

17. Morris, J.B., Wilkie, W.S., and Shusterman, D.J. 2005. Acute respiratory responses of the mouse to chlorine. Toxicol. Sci. 83:380-387.

18. Sawada, Y., Hosokawa, H., Matsumura, K., and Kobayashi, S. 2008. Activation of transient recep- tor potential ankyrin 1 by hydrogen peroxide. Eur. J. Neurosci. 27:1131-1142.

19. Andersson, D.A., Gentry, C., Moss, S., and Bevan, S. 2008. Transient receptor potential A1 is a sensory receptor for multiple products of oxidative stress. J. Neurosci. 28:2485-2494.

20. Mizushima, T., et al. 2006. Noxious cold stimulation induces mitogen-activated protein kinase activation in transient receptor potential (TRP) channels TRPA1- and TRPM8-containing small sensory neurons. Neuroscience. 140:1337-1348.

21. Oortgiesen, M., Veronesi, B., Eichenbaum, G., Kiser, P.F., and Simon, S.A. 2000. Residual oil fly ash and charged polymers activate epithelial cells and nociceptive sensory neurons. Am. J. Physiol. Lung Cell Mol. Physiol. 278:L683-L695.

22. Bandell, M., et al. 2004. Noxious cold ion channel TRPA 1 is activated by pungent compounds and bradykinin. Neuron. 41:849-857.

23. Dai, Y., et al. 2007. Sensitization of TRPA1 by PAR2 contributes to the sensation of inflammatory pain. J. Clin. Invest. 117:1979-1987.

24. Wang, S., et al. 2008. Phospholipase C and protein kinase A mediate bradykinin sensitization of TRPA1: a molecular mechanism of inflammatory pain. Brain. 131:1241-1251.

25. Taylor-Clark, T.E., et al. 2008. Prostaglandininduced activation of nociceptive neurons via direct interaction with transient receptor potential A1 (TRPA1). Mol. Pharmacol. 73:274-281.

26. Lee, L.Y., and Undem, B.J. 2005. Bronchopulmonary vagal afferent nerves. In Advances in vagal afferent neurobiology. B.J. Undem and D. Weinreich, editors. CRC Press/Taylor \& Francis. Boca-Raton, Florida, USA. 279-313.

27. Liedtke, W., et al. 2000. Vanilloid receptor-related osmotically activated channel (VR-OAC), a candidate vertebrate osmoreceptor. Cell. 103:525-535.

\title{
Measles virus breaks through epithelial cell barriers to achieve transmission
}

\section{Makoto Takeda}

\author{
Department of Virology, Faculty of Medicine, Kyushu University, Fukuoka, Japan.
}

\begin{abstract}
Measles is a highly contagious disease that causes immunosuppression in patients. Measles virus infection has been thought to begin in the respiratory epithelium and then spread to lymphoid tissue. In this issue of the JCI, Leonard et al. provide data to suggest an alternative model of measles virus pathogenesis (see the related article beginning on page 2448). In human primary epithelial cells and rhesus monkeys in vivo, the authors show that initial infection of respiratory epithelium is not necessary for the virus to enter the host but that viral entry into epithelial cells via interaction of the virus with a receptor located on the basolateral side of the epithelium is required for viral shedding into the airway and subsequent transmission.
\end{abstract}

Measles is an acute, viral infectious disease characterized by high fever, cough,

Nonstandard abbreviations used: CDV, canine distemper virus; $\mathrm{F}$, fusion (protein); $\mathrm{H}$, hemagglutinin (protein); MV, measles virus; SLAM, signaling lymphocytic activation molecule.

Conflict of interest: The author has declared that no conflict of interest exists.

Citation for this article: J. Clin. Invest. 118:2386-2389 (2008). doi:10.1172/JCI36251. and a maculopapular rash. Measles causes temporary and severe immunosuppression in patients, and secondary bacterial infections are a major cause of measles-related deaths (1). Studies have clearly shown that measles virus (MV), the causative agent, mainly replicates in lymphoid organs and causes devastating damage to the immune system of infected individuals (1). Despite the availability of effective vaccines, measles is still responsible for about $4 \%$ of deaths among children under 5 years of age worldwide (2). Importantly, the disease is transmitted via respiratory aerosols and is highly contagious in nature (1). In this issue of the JCI, Leonard et al. (3) report their detailed analyses of the interaction of MV with human host cells, in addition to data regarding MV pathogenesis in a rhesus monkey model. Their studies have revealed an elegant strategy by which MV propagates in patients and is transmitted to other target individuals and provide a good explanation for the highly contagious nature of measles.

\section{MV targets the host immune system}

$\mathrm{MV}$ is an enveloped virus possessing a nonsegmented negative-strand RNA genome and is classified into the Morbillivirus genus 


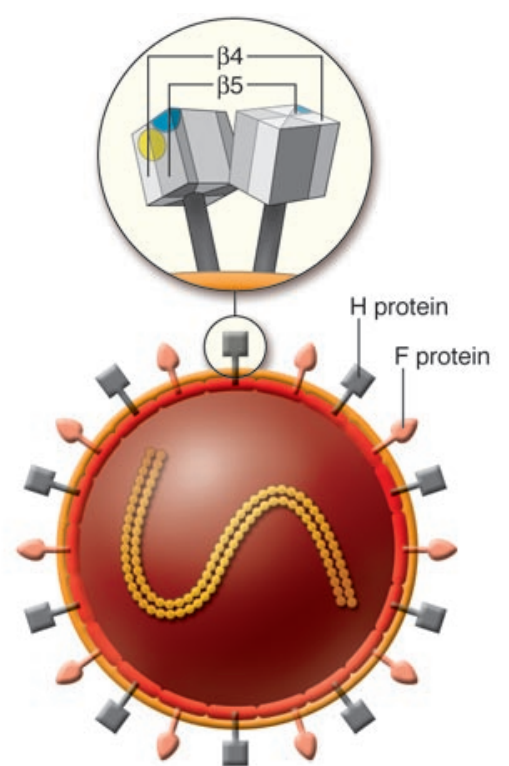

Measles virus

\section{Figure 1}

Schema of the MV particle and its receptor-binding $\mathrm{H}$ protein. MV is an enveloped virus and possesses 2 types of glycoprotein spikes, designated $\mathrm{H}$ and $\mathrm{F}$ proteins. The $\mathrm{F}$ protein mediates membrane fusion between the viral envelope and the host cell plasma membrane, while the $\mathrm{H}$ protein that forms homodimers on the viral envelope is responsible for binding of virus to receptors on target cells. The head domain of the $\mathrm{H}$ protein exhibits a six-bladed $\beta$-propeller fold. Residues important for using SLAM as a receptor map to a localized area of the $\beta 5$ sheet, forming a putative SLAM-binding site (shown in blue). On the other hand, relevant residues for epithelial cell infection map to a small area in the $\beta 5$ and $\beta 4$ sheets, forming another putative receptor-binding site (yellow) that is essentially distinct from the SLAM-binding site. of the Paramyxoviridae family (1). The virus has two types of glycoprotein spikes, designated hemagglutinin $(\mathrm{H})$ and fusion $(\mathrm{F})$ proteins, on the virus envelope (Figure 1). The $\mathrm{H}$ protein binds to specific molecules (receptors) on target cells, while the F protein mediates membrane fusion between the virus envelope and the host cell plasma membrane by cooperating with the $\mathrm{H}$ pro- tein (1). The H protein is a key determinant of MV cell tropism, since its binding to receptors is critical for MV to initiate its infectious cycle (4). In 2000, signaling lymphocytic activation molecule (SLAM; also known as CD150) was identified as a receptor for MV (5). SLAM is expressed on cells of the immune system, such as activated lymphocytes and dendritic cells (6). These findings could explain the lymphotropism and immunosuppressive properties of MV (4). Although the ubiquitously expressed CD46 molecule has been identified as an $\mathrm{MV}$ receptor, this molecule only functions as a receptor for laboratory-adapted vaccine strains of MV, and clinical MV strains do not use this molecule as a receptor (4). The use of SLAM as a receptor has been shown
A

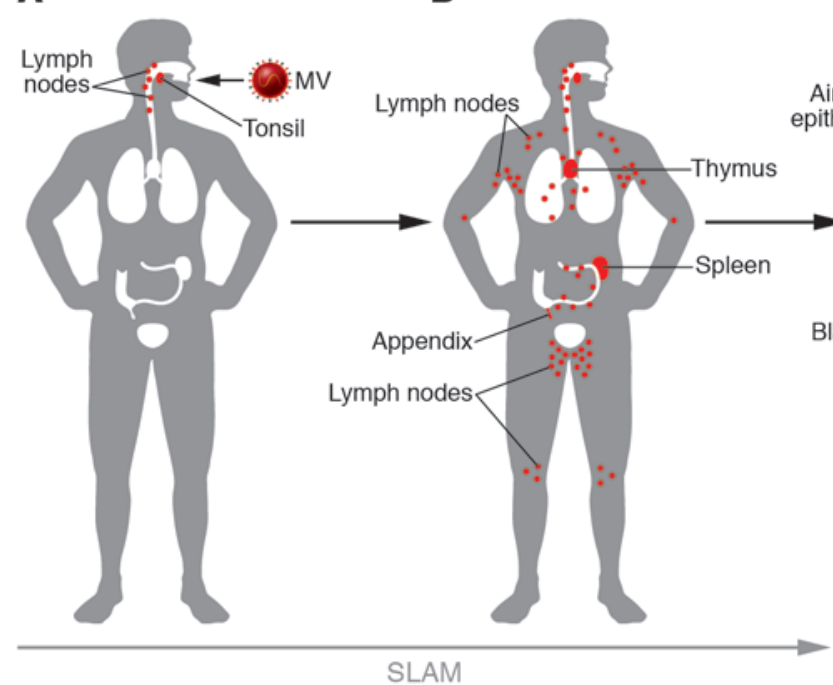

C

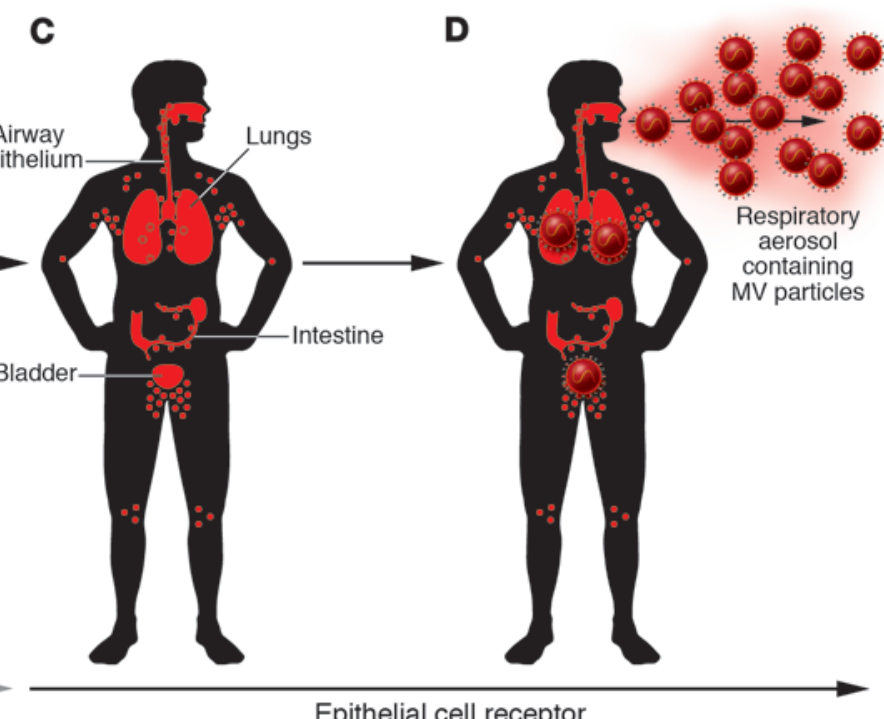

Figure 2

The time course of MV infection and receptor usage. (A) MV enters humans through the respiratory route and initiates its infectious cycle in lymphoid organs in the upper respiratory tract by using SLAM as a receptor. (B) MV-infected lymphocytes enter the bloodstream, and MV propagates in lymphoid organs throughout the body. (C) MV-infected immune cells appear to transmit MV to epithelial cells in various organs (e.g., airway, intestine, bladder). A putative epithelial cell receptor appears to play an important role in MV infection of epithelial cells. (D) MV then replicates in epithelial cells and actively releases progeny viruses into the airway. Consequently, respiratory aerosols of patients contain large amounts of MV particles. 
A
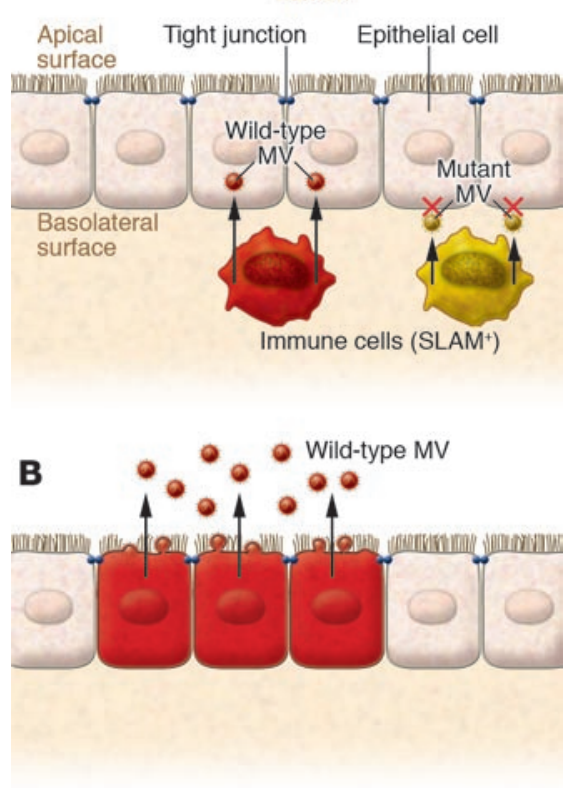

Figure 3

Model of MV shedding into the external surface of epithelium. (A) As Leonard et al. report in their current study (3), wild-type MV can be transmitted from infected immune cells (red) to epithelial cells via an epithelial cell receptor located on the basolateral surface of the latter. Mutant MV (yellow) that lacks the ability to use epithelial cell receptors fails to infect epithelial cells. The unidentified receptor may be a molecule related to tight junctions. (B) Progeny MV particles bud exclusively from the apical surface of the epithelium and are thus actively released into the airway. to be common among species of the Morbillivirus genus (7). Canine distemper virus (CDV) is a member of the Morbillivirus genus and causes a disease similar to measles in dogs. By generating a recombinant CDV that lacks the ability to bind SLAM, von Messling et al. (8) previously demonstrated that the ability to bind this receptor is crucial for CDV spread and virulence in vivo. Recombinant MVs engineered to express enhanced GFP are powerful tools for monitoring MV infection of cultured cells and animals (9). Using such viruses in a monkey model, de Swart et al. (10) reported that SLAM-positive lymphocytes and dendritic cells are the predominant cell types infected by MV in vivo. The data regarding recombinant viruses along with classic observations of MV pathology (11) clearly indicate that MV, by using SLAM as a receptor, primarily targets cells of the immune system to spread efficiently throughout the body of infected humans (Figure 2).

\section{Does MV infect epithelial cells?}

Histopathological data from patients with measles and monkeys experimentally infected with MV have indicated that although lymphoid organs are the major targets of MV, MV antigens and syncytia typical of MV-infected cells are also present in epithelial tissues of various organs (for references, see ref. 12). Primary cultures of epithelial and endothelial cells have also been shown to be susceptible to MV infection to some extent $(13,14)$. However, the mechanism by which
MV infects epithelial cells has remained unknown largely due to the lack of cell lines supporting MV infection independent of SLAM. In this issue of the JCI, Leonard et al. (3) have provided convincing evidence that MV has the ability to infect certain epithelial cell lines via a SLAM-independent mechanism. The authors demonstrated that three amino acid residues - leucine, proline, and tyrosine at amino acid positions 482, 497, and 543 , respectively - in the $\mathrm{H}$ protein are critical for MV infection of epithelial cells but not involved in MV infection of SLAMpositive immune cells. The crystal structure of the MV H protein determined in 2007 (15) has provided useful information regarding the $3 \mathrm{D}$ locations of each amino acid residue of the MV H protein. Mapping of the abovementioned residues in the crystal structure of the $\mathrm{H}$ protein, in this (3) and another recent study (16), clearly shows that the binding site for the epithelial cell receptor is located at a position different from that for SLAM (Figure 1). These data conclusively demonstrate that MV has an intrinsic ability to infect certain epithelial cells and immune cells via two distinctive mechanisms.

\section{MV emerges by breaking through epithelial barriers}

MV infection has long been thought to begin in the respiratory epithelium and then spread to lymphoid tissue. By intranasally inoculating monkeys with a recombinant mutant MV that retains the ability to use SLAM but lacks the ability to infect epithe- lial cells, Leonard et al. (3) demonstrated that the ability to infect epithelial cells is not required for MV to enter and spread in the host. Thus, their data support the idea that respiratory epithelial cells are not initial targets of MV $(4,17)$. Then what is the relevance of MV infection of epithelial cells in measles pathogenesis? Several tight junction or other cell junction molecules have been shown to be involved in the entry processes of various viruses, such as hepatitis C virus (18), reovirus (19), and herpes simplex virus (20). Leonard et al. (3) demonstrated that MV enters primary cultures of well-differentiated airway epithelial cells basolaterally and buds exclusively from the apical (luminal) side (Figure 3). Since the external surfaces of organs or tissues are composed of polarized epithelial cell layers, the ability of MV to infect these cells and bud from the apical side may facilitate virus shedding. Indeed, Leonard et al. (3) demonstrated in their rhesus monkey model that the mutant MV that cannot infect epithelial cells is not shed into the airway. MV therefore seems to infect epithelial cells in order to release progeny particles by breaking through the epithelial barrier (Figures 2 and 3). This active virus shedding into the airway may explain why MV is transmitted efficiently via respiratory aerosols, whereas human immunodeficiency virus, which shares with MV a similar tropism for immune cells, requires sexual contact or direct infusion into blood for transmission. 


\section{Perspectives}

The study by Leonard et al. (3) and other recent work $(12,15,16)$ have greatly advanced our understanding of the molecular bases of the pathology of measles. However, an important piece of information is still missing. What is the MV receptor on epithelial cells? Data obtained by Leonard et al. (3) and Tahara et al. (16) suggest that the receptor may be a molecule related to tight junctions. Use of cell junction molecules as receptors may be a common strategy for viruses to facilitate their transmission (18-20). Identification in future studies of the epithelial cell receptor for MV will likely reveal further details of the elegant strategy of infection (i.e., differential usage of two receptors during the course of infection) employed by this highly contagious virus.

\section{Acknowledgments}

The author would like to thank Yusuke Yanagi for his critical reading of the manuscript and helpful suggestions.

Address correspondence to: Makoto Takeda, Department of Virology, Faculty of Medicine, Kyushu University, Fukuoka
812-8582, Japan. Phone: 81-92-642-6138; Fax: 81-92-642-6140; E-mail: mtakeda@ virology.med.kyushu-u.ac.jp.

1. Griffin, D.E. 2007. Measles virus. In Fields virology. D.M Knipe et al., editors. Lippincott Williams \& Wilkins. Philadelphia, Pennsylvania, USA. pp. 1551-1585.

2. Bryce, J., Boschi-Pinto, C., Shibuya, K., and Black, R.E. 2005. WHO estimates of the causes of death in children. Lancet. 365:1147-1152.

3. Leonard, V.H.J., et al. 2008. Measles virus blind to its epithelial cell receptor remains virulent in rhesus monkeys but cannot cross the airway epithelium and is not shed. J. Clin. Invest. 118:2448-2458.

4. Yanagi, Y., Takeda, M., and Ohno, S. 2006. Measles virus: cellular receptors, tropism and pathogenesis. J. Gen. Virol. 87:2767-2779.

5. Tatsuo, H., Ono, N., Tanaka, K., and Yanagi, Y. 2000. SLAM (CDw150) is a cellular receptor for measles virus. Nature. 406:893-897.

6. Veillette, A. 2006. Immune regulation by SLAM family receptors and SAP-related adaptors. Nat. Rev. Immunol. 6:56-66.

7. Tatsuo, H., Ono, N., and Yanagi, Y. 2001. Morbilliviruses use signaling lymphocyte activation molecules (CD150) as cellular receptors. J. Virol. 75:5842-5850.

8. von Messling, V., Svitek, N., and Cattaneo, R. 2006. Receptor (SLAM [CD150]) recognition and the V protein sustain swift lymphocyte-based invasion of mucosal tissue and lymphatic organs by a morbillivirus. J. Virol. 80:6084-6092.

9. Hashimoto, K, et al. 2002. SLAM (CD150)-independent measles virus entry as revealed by recombinant virus expressing green fluorescent protein. J. Virol. 76:6743-6749.

10. de Swart, R.L., et al. 2007. Predominant infection of
CD150(+) lymphocytes and dendritic cells during measles virus infection of macaques. PLoS Pathog. 3:e178.

11. Nii, S., et al. 1964. Experimental pathology of measles in monkeys. Biken J. 6:271-297.

12. Takeda, M., et al. 2007. A human lung carcinoma cell line supports efficient measles virus growth and syncytium formation via a SLAM- and CD46independent mechanism. J. Virol. 81:12091-12096.

13. Takeuchi, K., Miyajima, N., Nagata, N., Takeda, M., and Tashiro, M. 2003. Wild-type measles virus induces large syncytium formation in primary human small airway epithelial cells by a SLAM(CD150)independent mechanism. Virus Res. 94:11-16.

14. Andres, O., et al. 2003. CD46- and CD150-independent endothelial cell infection with wild-type measles viruses. J. Gen. Virol. 84:1189-1197.

15. Hashiguchi, T., et al. 2007. Crystal structure of measles virus hemagglutinin provides insight into effective vaccines. Proc. Natl. Acad. Sci. U. S. A. 104:19535-19540.

16. Tahara, M., et al. 2008. Measles virus infects both polarized epithelial and immune cells using distinctive receptor-binding sites on its hemagglutinin. J. Virol. 82:4630-4637.

17. de Witte, L., et al. 2008. DC-SIGN and CD150 have distinct roles in transmission of measles virus from dendritic cells to T-lymphocytes. PLoS Pathog. 4:e1000049.

18. Evans, M.J., et al. 2007. Claudin-1 is a hepatitis C virus co-receptor required for a late step in entry. Nature. 446:801-805.

19. Barton, E.S., et al. 2001. Junction adhesion molecule is a receptor for reovirus. Cell. 104:441-451.

20. Geraghty, R.J., et al. 1998. Entry of alphaherpesviruses mediated by poliovirus receptor-related protein 1 and poliovirus receptor. Science. 280:1618-1620.

\title{
Mechanisms of resistance to ErbB-targeted cancer therapeutics
}

\section{Qiang Wang and Mark I. Greene}

Department of Pathology and Laboratory Medicine, University of Pennsylvania School of Medicine, and Abramson Family Cancer Research Institute, Philadelphia, Pennsylvania, USA.

\begin{abstract}
The ErbB receptors, such as EGFR, have been intensely pursued as targets for cancer therapeutics. However, a large percentage of patients who are initially responsive to ErbB-targeted therapies experience tumor recurrence and become refractory to therapy. In this issue of the JCI, Guix et al. demonstrate that downregulation of IGF-binding protein 3 (IGFBP-3) and -4 , the negative regulators of IGF-I receptor signaling, contributes to the resistance of human squamous cell carcinomas to the EGFR inhibitor gefitinib (see the related article beginning on page 2609). Understanding the mechanisms involved in the resistance of some tumors to ErbB-targeted molecules may provide guidelines for developing more efficient therapeutic approaches.
\end{abstract}

Nonstandard abbreviations used: GR, gefitinib resistant; IGFBP, IGF-binding protein; IGF-IR, IGF-1 receptor; $\mathrm{mTOR}$, mammalian target of rapamycin; TKI, tyrosine kinase inhibitor.

Conflict of interest: The authors have declared that no conflict of interest exists.

Citation for this article: J. Clin. Invest. 118:2389-2392 (2008). doi:10.1172/JCI36260.
Members of the ErbB family of receptor tyrosine kinases, which include EGFR, ErbB2 (known as $\mathrm{p} 185^{\mathrm{c} \text {-neu }}$ in rodents and HER2 in humans), ErbB3, and ErbB4, are overexpressed in a variety of human solid tumors (1). Activation of the ErbB molecules correlates strongly with the pathogenesis and poor prognosis of many forms of cancer. Ligands for EGFR, ErbB3, and ErbB4, such as EGF and the polypeptide heregulin, bind to the extracellular domain of the receptor, leading to receptor dimerization and autophosphorylation of the intracellular tyrosine kinase domain. These events subsequently upregulate downstream signaling cascades, including the MAPK, PI3K, and mammalian target of rapamycin (mTOR) pathways. As such, ErbB molecules modulate cell proliferation, survival, and mobility.

\section{ErbB-targeted cancer therapeutics}

For the past twenty years, targeting the ErbB receptors has been intensely pursued as an important cancer therapeutic strategy (1). Immunological approaches have 